\title{
Development of a Displacement- and Frequency-Noise-Free Interferometer in a 3D Configuration for Gravitational Wave Detection
}

\author{
Keiko Kokeyama, ${ }^{1, *}$ Shuichi Sato, ${ }^{2}$ Atsushi Nishizawa, ${ }^{3}$ Seiji Kawamura, ${ }^{4}$ Yanbei Chen, ${ }^{5}$ and Akio Sugamoto ${ }^{6}$ \\ ${ }^{1}$ Graduate School of Humanities and Sciences, Ochanomizu University, 2-1-1, Otsuka, Bunkyo-ku, Tokyo 112-8610 Japan \\ ${ }^{2}$ Faculty of Engineering, Hosei University, 3-7-2, Kajino-cho, Koganei, Tokyo 184-8584 Japan \\ ${ }^{3}$ Graduate School of Human and Environmental Studies, Kyoto University, Kyoto 606-8501 Japan \\ ${ }^{4}$ TAMA project, National Astronomical Observatory of Japan, 2-21-1, Mitaka, Osawa, Tokyo 181-8588 Japan \\ ${ }^{5}$ Theoretical Astrophysics, California Institute of Technology, Pasadena, California 91125 USA \\ ${ }^{6}$ Ochanomizu University, 2-1-1, Otsuka, Bunkyo-ku, Tokyo 112-8610 Japan
}

(Received 5 June 2009; published 22 October 2009)

\begin{abstract}
The displacement- and frequency-noise-free interferometer (DFI) is a multiple laser interferometer array for gravitational-wave detection free from both the displacement noise of optics and laser frequency noise. So far, partial experimental demonstrations of the DFI have been done in 2D table top experiments. In this Letter, we report the complete demonstration of a 3D DFI. The DFI consists of four Mach-Zehnder interferometers with four mirrors and two beam splitters The attained maximum suppression of the displacement noise of both mirrors and beam splitters was $40 \mathrm{~dB}$ at about $50 \mathrm{MHz}$. The nonvanishing DFI response to a gravitational wave was successfully confirmed using multiple electro-optic modulators and computing methods.
\end{abstract}

DOI: 10.1103/PhysRevLett.103.171101

PACS numbers: 04.80.Nn, 06.30.Ft, 95.55.Ym

Gravitational waves are perturbations of the space-time curvature propagating across the Universe at the speed of light. They propagate in space-time, extending a proper distance between test masses in one transverse direction, and shortening in the other, orthogonal direction. A laserinterferometric gravitational-wave detector measures length differences between the test masses caused by the gravitational-wave effect. Various noise sources that limit the sensitivity of the detector must be removed to achieve the best sensitivity level, as the interferometers must detect the very tiny signals of length change. The sensitivities of the ground-based detectors are affected by seismic noise, thermal noise, radiation pressure noise, laser source noise (laser frequency and intensity noise), and shot noise. The seismic, thermal and radiation pressure noises can be categorized as displacement noises since the noise sources shake up the optics directly. These displacement noises limit the sensitivities especially in the low-frequency regime, which is the important region for the ground-based gravitational-wave detectors, which target at several ten to several thousand Hz. Recently, the idea of a gravitationalwave detector free from both the displacement noises of the optics and the laser frequency noises at all frequencies has been proposed [1,2]. The displacement- and frequencynoise-free interferometer (DFI) technique exploits the fact that the gravitational-wave effect on the laser light takes a form different from that of optics displacements. A signal combination which does not sense displacement or frequency noises but senses the gravitational-wave contribution can be constructed when the array of $N$ test masses consists of multiple interferometers under the condition that $N>d+2$ ( $d$ is the number of spatial dimensions of the array). In principle, the DFI technique can remove all the displacement and frequency noises and accomplish the shot-noise limited sensitivity at all frequencies. This means that if we can properly incorporate the DFI technique into the 2nd generation detectors such as Advanced LIGO [3], Advanced Virgo [4], LCGT [5], GEO-HF [6], and AIGO [7], we could attain a strain sensitivity of $4 \times 10^{-24} \mathrm{~Hz}^{-1 / 2}$ even much below $100 \mathrm{~Hz}$. This will significantly improve the inspiral range especially for heavier-mass binaries. It should be noted, however, that in the DFI configurations proposed so far the shot-noise limited sensitivity itself is impaired at lower frequencies because the gravitationalwave signals are canceled in the process of eliminating the noises. To circumvent this problem, other advanced configurations with optical cavities have been studied [8-11], and further efforts should be made to make the DFI technique applicable to the ground-based detectors.

In this Letter, we report the displacement noise suppression of interferometer optics while the gravitational-wave response is retained using the DFI configuration suggested by Ref. [12]. Although there has been only a proof-ofprinciple experiment with a 2D layout of a subpart of this configuration [13] so far, our experiment is the first demonstration with a complete DFI configuration. In our configuration, the gravitational-wave sensitivity deteriorates in the low frequencies in proportion to $\Omega^{2}$ with $\Omega$, the gravitational-wave frequency. It is the best sensitivity of current proposed configurations.

The 3D DFI configuration is depicted in Fig. 1. Four Mach-Zehnder interferometers (MZIs) with equal arm lengths compose the DFI. Let us denote by $\mathrm{IFO}_{1}, \mathrm{IFO}_{2}$, $\mathrm{IFO}_{3}$, and $\mathrm{IFO}_{4}$ the four single MZIs drawn in orange 


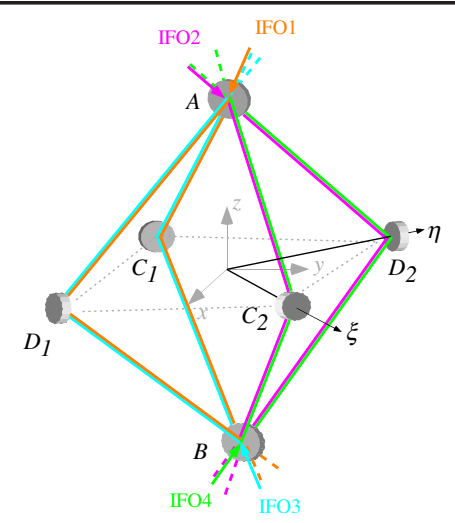

FIG. 1 (color online). Optical topology of 3D DFI. The pairs of $\mathrm{IFO}_{1}$ and $\mathrm{IFO}_{3}$ and of $\mathrm{IFO}_{2}$ and $\mathrm{IFO}_{4}$ are counterpropagating to each other, constructing two bidirectional Mach-Zehnder interferometers (MZIs). Right and left bidirectional MZIs share the two beam splitters.

$\left(A C_{1} B-A D_{1} B\right)$, pink $\left(A C_{2} B-A D_{2} B\right)$, light blue $\left(B C_{1} A-B D_{1} A\right)$, and green $\left(B C_{2} A-B D_{2} A\right)$ in Fig. 1. Each length between a beam splitter and a mirror $\left(A C_{1}\right.$, $A C_{2}, A D_{1}, A D_{2}, C_{1} B, C_{2} B, D_{1} B$, and $\left.D_{2} B\right)$ is assumed as $L$ so that each interferometer is insensitive to laser frequency noise thanks to the equal arms. Two of the four interferometers are combined to construct one bidirectional MZI in which two beams are counterpropagating on the same path. As is discussed in Ref. [12], the DFI signal combination can be written as

$$
\phi_{\mathrm{DFI}}=\left(\phi_{1}-\phi_{3}\right)-\left(\phi_{2}-\phi_{4}\right)
$$

where $\phi_{j}(j=1,2,3,4)$ are the phase signals of $\mathrm{IFO}_{j}$. In Eq. (1), the combined signal of $\left(\phi_{1}-\phi_{3}\right)$ is the output of the bidirectional MZI that is free from the displacements of $C_{1}$ and $D_{1}$. Because the folding mirrors are at the midpoint of the arms, and the laser beams travel in both directions in the MZI, the phase variation due to the displacement of $C_{1}$ and $D_{1}$ arrives at each output port simultaneously. Therefore, in the frequency domain, $\mathrm{IFO}_{1}$ and $\mathrm{IFO}_{3}$ have identical transfer functions from the displacement of $C_{1}$ and $D_{1}$ to the signal ports, expressed as

$$
H_{1}^{C_{1}, D_{1}}(\Omega)=H_{3}^{C_{1}, D_{1}}(\Omega)=e^{-i L \Omega / c},
$$

where $c$ is the speed of light in vacuum, and $\Omega$ the Fourier frequency of the displacement motion. The phase modulations due to the motions of the optics experience the same phase delay shown in Eq. (2) because the displacement sources are at the midpoint of the path. These identical responses to $C_{1}$ and $D_{1}$ can thus be removed by subtraction. Similarly, $\left(\phi_{2}-\phi_{4}\right)$ is the signal of the other pair of bidirectional MZIs, and is free from the displacements of $C_{2}$ and $D_{2}$. Any signals that phase modulate the light and do not arise at the exact center of the path will not arrive simultaneously at the outputs, and thus will not be canceled by the bidirectional MZIs. The beam splitter displacements are removed by the combination of $\left(\phi_{1}-\phi_{2}\right)$ in Eq. (1).
Since the laser beams of $\mathrm{IFO}_{1}$ and $\mathrm{IFO}_{2}$ reach the two beam splitters simultaneously, their responses to the beam splitter motion are the same. Therefore, in the frequency domain, $\mathrm{IFO}_{1}$ and $\mathrm{IFO}_{2}$ have identical transfer functions from the displacement of $A$ to the signal ports expressed as

$$
H_{1}^{A}(\Omega)=H_{2}^{A}(\Omega)=e^{-2 i L \Omega / c} .
$$

The transfer functions of the displacement motion of beam splitter $B$ are $H_{1}^{B}(\Omega)=H_{2}^{B}(\Omega)=1$ since the motions of $B$ are just before the detection ports. Therefore, the beam splitter displacements can be eliminated by the combination of $\mathrm{IFO}_{1}$ and $\mathrm{IFO}_{2}$. Similarly, the combined signal of $\left(-\phi_{3}+\phi_{4}\right)$ in Eq. (1) is free from the beam splitter displacements. In practice, in addition to the transfer functions, extra phase delays should be considered that arise from the paths from the second beam splitter to the signal analyzer via a photo detector and signal cables. These additional phase delays of each interferometer should be made equal or calibrated later, so as not to lose the balanced responses.

According to Ref. [12], the DFI signal responds to a gravitational wave with the transfer function,

$$
\begin{aligned}
H_{\mathrm{GW}}(\Omega)= & -\frac{i \omega}{\Omega}\{(1+1 / \sqrt{2}) \\
& \times e^{-(i \Omega L / \sqrt{2} c)}\left(1-e^{-[2 i \Omega L(1-1 / \sqrt{2}) / c]}\right) \\
& \left.-(1-1 / \sqrt{2}) e^{(i \Omega L / \sqrt{2} c)}\left(1-e^{-[2 i \Omega L(1+1 / \sqrt{2}) / c]}\right)\right\}
\end{aligned}
$$

where $\Omega$ is the Fourier frequency of the gravitational wave, $\omega$ is the laser frequency, and we assume the gravitational wave of $\eta-\xi$ polarization is coming along the $z$ direction in Fig. 1. The 3D DFI does not respond to DC gravitational waves because the response is degraded by the subtraction when the DFI signal is built by the four MZI signals. The DFI response is proportional to $(\Omega L / c)^{2}$ below the peak frequency. The peak frequency depends on the arm length $L$, e.g., when $L=1 \mathrm{~m}$, the peak is at about $150 \mathrm{MHz}$.

Figure. 2. shows the practical setup of our experiment. The laser source is a commercial solid-state Nd:YAG laser at $1064 \mathrm{~nm}$. The light source is split into four by beam splitters so as to provide the four incident beams for the four MZIs. As depicted in Fig. 2, $\mathrm{IFO}_{2}$ and $\mathrm{IFO}_{4}$ (denoted as higher interferometers) are lying over $\mathrm{IFO}_{1}$ and $\mathrm{IFO}_{3}$ (denoted as lower interferometers). The length $L$ was chosen to be about $40 \mathrm{~cm}$ yielding a peak frequency of about $370 \mathrm{MHz}$. There are two Faraday isolators before the lasers are sent to $\mathrm{IFO}_{1}$ and $\mathrm{IFO}_{2}$. They are used for extracting the signal of $\mathrm{IFO}_{3}$ and $\mathrm{IFO}_{4}$ at ports $t t 3$ and $t t 4$ exiting from the $\mathrm{IFO}_{1}$ and $\mathrm{IFO}_{2}$ inputs, respectively. The signals at ports $t r 1$ and $t r 2$ are directly detected by the photo detectors. The DC photo detectors (DCPD) are placed at ports $t r 3$ and $t r 4$ for extracting the control signals. The extracted DC signals are sent to the servo filters with static offsets, then sent to the piezoelectric 


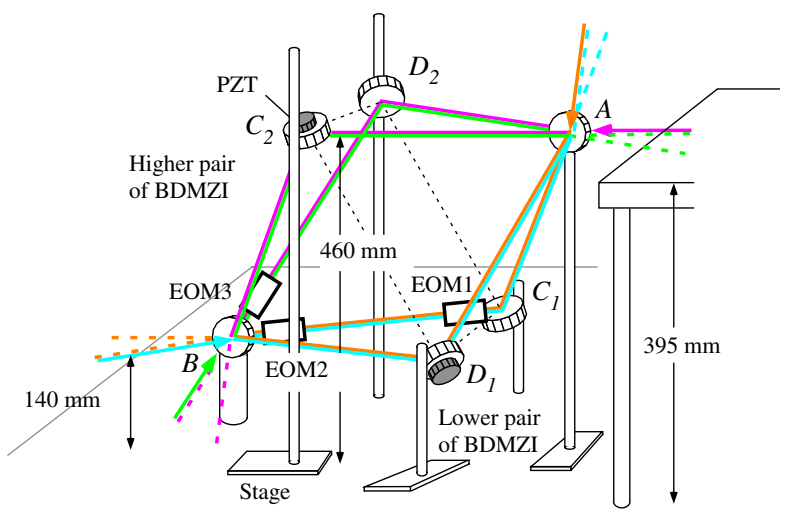

FIG. 2 (color online). Schematic of the experiment. The octahedron is pushed over sideways to be set up on an optical table. $\mathrm{IFO}_{1}$ and $\mathrm{IFO}_{3}$ comprise one bidirectional MZI, and so do $\mathrm{IFO}_{2}$ and $\mathrm{IFO}_{4}$. EOM1 simulates mirror $C_{1}$ displacement and EOM2 and EOM3 simulate beam splitter $B$ displacement.

transducers (PZTs) attached to mirrors $D_{1}$ and $C_{2} . D_{1}$ and $C_{2}$ are controlled by signals giving the midfringe locking for the $\mathrm{IFO}_{3}$ and $\mathrm{IFO}_{4}$, respectively. Once $\mathrm{IFO}_{3}$ is locked, $\mathrm{IFO}_{1}$ is locked automatically. Likewise, locking $\mathrm{IFO}_{2}$ automatically locks $\mathrm{IFO}_{4}$ The control bandwidth of the two interferometers is about $1 \mathrm{kHz}$.

To align the four-interferometer system, we utilized an optical lever as shown in Fig. 3, using the fact that the lower and higher sets of bidirectional MZIs share their beam splitters [14]. The positions and the angles of $C_{1}$ and of $D_{1}$, and positions and angles of $C_{2}$ and of $D_{2}$, independently determine the position and angle of $B$ for the alignment of the higher and lower interferometer, respectively. To make them compatible, the optical lever was utilized to monitor the angle of beam splitter $B$. First, for the lower interferometer, the angle of $B$ determined by $C_{1}$ and $D_{1}$, is adjusted and monitored by the optical lever signal on the screen. For the higher interferometer, we then adjust the position and angle of $B$ determined by $C_{2}$ and $D_{2}$, is adjusted for the lower interferometer and displayed on the screen. And we adjust the positions and angles of $C_{2}, D_{1}$ and $A$ so that the two monitored marks on the screen come to exactly the same point which means the ideal angle of $B$. This method achieved an almost perfect contrast.

Electro-optic modulators (EOMs) were utilized to create phase shifts to the laser as simulated displacements of the optics because the interesting frequency, i.e., the response peak of the gravitational wave, is expected to be in the hundred-MHz region. To confirm the noise cancellation features of both mirror and beam splitter, the three EOMs were driven; for simulating the displacement of mirror $C_{1}$, EOM1 is placed at the center of the path $B C_{1} A$; for

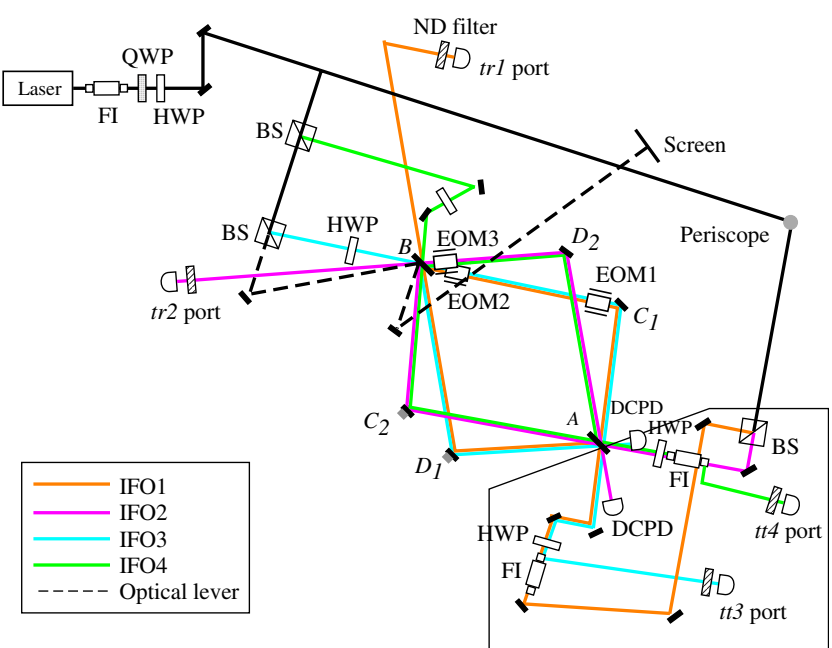

FIG. 3 (color online). The overhead view of the experimental setup. HWP: half wave plate, QWP: quarter wave plate, FI: Faraday islator, BS: beam splitter, DCPD: DC photo detector. $\mathrm{IFO}_{1}\left(A-C_{1}, D_{1},-B\right), \mathrm{IFO}_{2}\left(A-C_{2}, D_{2},-B\right), \mathrm{IFO}_{3}\left(B-C_{1}\right.$, $\left.D_{1},-C\right)$ and $\mathrm{IFO}_{4}\left(B-C_{2}, D_{2},-A\right)$. The black-dashed line indicates the optical lever method for the alignment of $3 \mathrm{D}$ configuration.

simulating the displacements of beam splitter $B$, EOM2 and EOM 3 are placed close to $B$ as beam splitter $B$ affects both the higher and lower interferometer. The EOMs were driven by swept-sine noise sources provided by the $\mathrm{rf}$ network analyzer (Agilent 4395A). The signals from the four MZIs are detected by four fast photo detectors (New Focus 1611) at ports $t r 1, t r 2, t t 3$ and $t t 4$. These four signals are electrically combined so as to form the DFI signal shown in Eq. (1). We adjusted the control polarity of the MZIs so that $\operatorname{tr} 1$ and $\operatorname{tr} 2$ have opposite sign to cancel by their summation. The optical gain imbalances between the four signals are compensated by neutral density (ND) filters placed in front of each detector.

The DFI response to a gravitational wave was demonstrated by multiple measurements and simulation. Ideally, the continuous phase variation should be applied on the entirety of the laser paths to simulate the gravitationalwave effect. However, only the part covered by the crystal causes a phase shift by an EOM. Therefore, the following procedure was adopted. We place an EOM in the laser path and measure the transfer function from the EOM to the DFI signal. Then we place the EOM at the next point and measure the transfer function, and so on. The measured data are summed up with proper phase delays that depend on the gravitational-wave direction and the EOM position. With the summation of the discrete phase shifts, the DFI response shown in Eq. (4) can be approximated as

$$
H_{\mathrm{GW}}(\Omega) \sim H_{\mathrm{sum}}(\Omega)=\frac{\omega L}{c D}\left\{e^{(i \Omega L / \sqrt{2} c)} \sum_{n=0}^{2 D} e^{(i \Omega L / c)(n / D)} e^{-(i \Omega L / \sqrt{2} c)(n / D)}-e^{-(i \Omega L / \sqrt{2} c)} \sum_{n=0}^{2 D} e^{(i \Omega L / c)(n / D)} e^{(i \Omega L / \sqrt{2} c)(n / D)}\right\}
$$



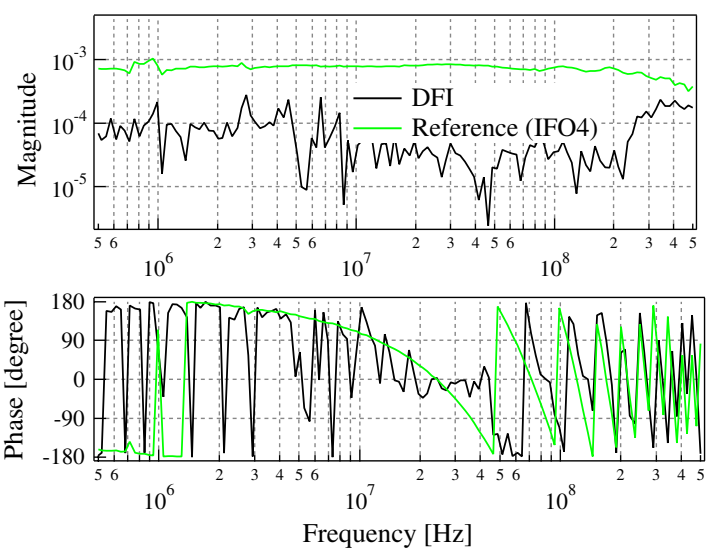

FIG. 4 (color online). The solid green line is the transfer function from EOM3 to $t t 4$ as a reference, and the solid black line is the transfer function from EOM3 to the DFI signal. In the DFI channel, from 1 to 2 orders of magnitude of the cancellation were attained from $500 \mathrm{kHz}$ to $200 \mathrm{MHz}$.

under the condition $\Omega L / c D \ll 1$, where the gravitational wave is assumed to come along the $A B$ axis. $D$ is the resolution of the positions on the path of $L$ where the phase shifts occur. Note that the straightforward way to produce continuous phase shift is to insert EOMs all along the laser path. However, that many EOMs covering laser paths will cause a serious reduction in interferometer contrast.

In the experiment, the phase shift part of $e^{i \Omega L n / D c}$ in Eq. (5) was created practically by the EOM with the discrete resolution $D=10$. For the first ten data, $n=$ $0 \ldots 9$, an EOM was set in a respective position at $n L / D$ from beam splitter $B$, and the respective transfer functions were measured. Since we cannot put an EOM on $C_{1} A$, we compute the transfer function data for $n=10 \ldots 20$ applying proper phase delays to the data of $n=9$. These data were summed with predicted phase delays due to the incident angle of the gravitational wave in accordance with Eq. (5), to construct the gravitational wave response.

The results for displacement-noise suppression are shown in Fig. 4. The solid black line is the transfer function from EOM1 to the DFI (combined) signal. The solid green line is the transfer function from EOM1 to a single MZI output (port $t t 4$ ) as a reference. Comparing with these transfer functions, we can confirm that the DFI signal has less sensitivity to the displacement by 1 to 2 orders of magnitude in a wide frequency band from $500 \mathrm{kHz}$ to $200 \mathrm{MHz}$. The transfer functions in Fig. 4. include the response of the EOMs and the photo detectors, and also phase delays due to the optical and signal paths after the photo detectors to the analyzer. In Fig. 5, the orange dots are the simulated DFI response to a gravitational wave obtained by the measurement and computing, and the gray solid line is the DFI response predicted by Eq. (4). The response function of photo detectors, and of the me-
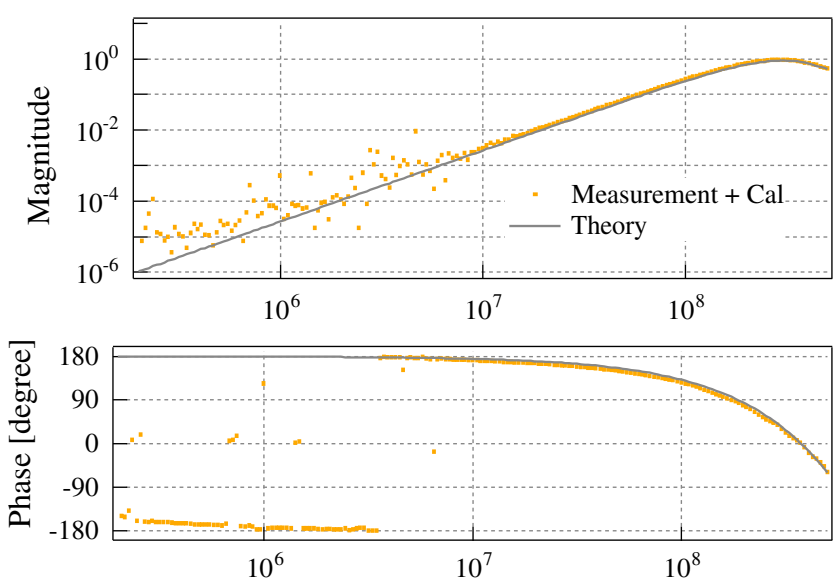

Frequency $[\mathrm{Hz}]$

FIG. 5 (color online). The orange dots are the simulated DFI response to the gravitational wave obtained by being measured and computed, the gray solid line is the DFI response predicted by Eq. (4). The simulated response agreed with the theory.

chanical resonance due to the EOM crystal, and the phase delays due to the optical paths and signal cables from beam splitters to the analyzer are compensated properly in Fig. 5. The 3D DFI response to a gravitational wave is well demonstrated around the peak frequency and its $\Omega^{2}$ feature can be confirmed just below that frequency.

We thank for Grant-in-Aid of the Japan Society for the Promotion of Science and Mitsubishi Foundation. This research is partially supported by the U.S. National Science Foundation.

*keiko.kokeyama@nao.ac.jp

[1] S. Kawamura and Y. Chen, Phys. Rev. Lett. 93, 211103 (2004).

[2] Y. Chen and S. Kawamura, Phys. Rev. Lett. 96, 231102 (2006).

[3] LIGO Scientific Collaboration, Classical Quantum Gravity 26, 114013 (2009).

[4] http://wwwcascina.virgo.infn.it/advirgo/

[5] Kazuaki Kuroda and LCGT Collaboration, Classical Quantum Gravity 23, S215 (2006).

[6] B. Willke et al., Classical Quantum Gravity 23, S207 (2006).

[7] D. G. Blair et al., J. Phys. Conf. Ser. 122, 012001 (2008).

[8] S.P. Tarabrin and S. P. Vyatchanin, Phys. Lett. A 372, 6801 (2008).

[9] A. A. Rakhubovsky and S.P. Vyatchanin, Phys. Lett. A 373, 13 (2008).

[10] A. Nishizawa et al., Phys. Rev. Lett. 101, 081101 (2008).

[11] S.P. Vyatchanin, arXiv:0808.3445.

[12] Y. Chen et al., Phys. Rev. Lett. 97, 151103 (2006).

[13] S. Sato et al., Phys. Rev. Lett. 98, 141101 (2007).

[14] K. Kokeyama, Ph.D. thesis, Ohanomizu University, 2009. 